\title{
Study on Fiber Optics Distributed Acoustic Sensing Technology
}

\author{
Jianghe Liu, Siyuan Wei, Zhongxin Qi \\ Military Economics Academy of PLA, Wuhan City, Hubei Province, 430035, China
}

Key words: Distributed, Fiber-optics sensing technology, acoustic wave

\begin{abstract}
Distributed fiber optics sensing technology is a technology ofbroad application prospect. It allows a long distance and a wide range of acoustic emission source positioning and monitoring, while perform a real-time, online, dynamic monitoring of damage to the structural building materials, as well as the positioning of damage location. As to civil engineering, if the distributed fiber-optics sensor can be successfully used, it will then bring great economic benefits. In this paper, the author makes a general summary to the fiber optics distributed acoustic sensing technology by using the collected data.
\end{abstract}

\section{Main advantages/disadvantages of distributed fiber-opticsacoustic sensing technology}

The acoustic emission signal is a kind of elastic stress wave which is formed from the inside of the material due to the sudden release of the strain energy. It contains the information of the internal damage of the material, so the acoustic emission technology can be used to identify the different damages that occur in the loaded material. The basic principle of traditional acoustic emission (AE) detection is toexert pressure or other external conditions (e.g. high temperature, etc.) to the object to be detected, so that the defect or potential defect in such object is automatically sounded, and estimate the location and size of such defect according to the stress received from the defect. Also,the fiber-optics sensor substitutes the traditional piezoelectric ceramic-made sensor with the light and small fiber optics. Compared with the traditional piezoelectric ceramic PZT, fiber-optics AE sensor is characterized bywide frequency band, free from electromagnetic interference, high sensitivity, small size, high damage threshold, do not have to contact with the measured object, and with great spacing to electronic equipment and sensors, as well as other advantages.

Fiber-optics AE sensor is the most widely used phase modulation-type fiber-optics sensor. It works as follows: the AE wave acts on thesensitive part, making the refractive index or the propagation constant of the fiber optics change, which leads to the change of the phase of the light wave, and measure the external parameters by detecting the change of the optical phase. Because the current types of photodetectors cannot directly sense the phase change of optics, it is necessary to use the interfering technique to convert the phase change of optics into intensity change. Therefore, the phase modulation type sensor is also known as the interference-type sensor. Compared with other modulation methods, phase modulation has high detection sensitivity and wide dynamic detection range due to its application of interference technology, and the probe form is diverse, which is suitable for different detection environments. In consequence, most of the current fiber-optics AE detectionsemploy the modulated fiber-optics sensor.

Where the change in AE wave results in changes in the intensity of the fiber optics, you can measure the change in light intensity to detect the AE signal, which is known as intensity modulation. Intensity modulation is a method that is used earliest among fiber optic sensing technologies. It has advantage such as simple technology, low cost, and has a certain application in 
low-precision occasions requiring no AE source positioning. The disadvantage lies in that it is difficult to sense at forming point, and the light source fluctuations, connector loss and photodetector response changewill cause light intensity fluctuations, low sensitivity, and even impossible to detect weak AE signal. Moreover,the AE signal is generally very weak, and has fast attenuation in solid, so theintensity modulation is rarely used in actual AE detection, which is primarily used to measure the displacement, sound waves in audible range and other parameters.

Currently, the ways used to modulate the optical wavelength are mainly optical frequency-selecting and filtering. The traditional optical wavelength modulation methods are primarily the F-P interferometer filter, birefringence characteristic based filtering, various displacement spectrum selection and other external modulation technologies. In recent years, the rapid development of fiber grating filtering technology has opened up a new prospect forfunctional optical wavelength modulation. The wavelength modulation-type fiber-optic AE sensorswhich have been covered in more papers in the last few years are mainly FBG-type AE sensor, of which the sensitive component is FBG. It works as follows: pass through the Bragg wavelength of $\mathrm{AE}$-wave modulation grating, and then, detect the $\mathrm{AE}$ signal according to the change of reflected wavelength. BaldwinC $S$ et al. successfully applied the FBG sensor in simulating the AE-source AE signal detection. FBG sensor features high sensitivity, which is easy to form a distributed measurement, but it is sensitive to temperature, bringing complexity to demodulation program.

The AE sensor based on fiber optics coupler is a new type of fiber-optics AE sensor. Its working principle can be as follows: the acoustic wave acts on the fiber optics coupler, so that the fiber optics coupler output optical power changes, through which the nature of acoustic waves can be known. The sensor is advantageous for its simple principle, easy manufacturing, and is easy for mass production.

\section{Application of distributed fiber optics acoustic sensing technology}

Fiber optics hydrophone is one of the most important applications of fiber AE detection technology, which are primarily used in acoustic propagation in marine acoustic environment, submarine acoustic monitoring, target acoustic monitoring, ocean, terrestrial oil and gas exploration, marine and terrestrial seismic waves monitoring and marine environmental detecting. Also, it is an advanced detection measure for modern naval anti-submarine warfare and underwater weaponstesting. Cranch G A and Nash P pointed out that the fiber optics hydrophone array is developing in the direction of large-scale time-division and dense wavelength division multiplexing, to meet the needs of large-scale arraying in future sonar system.

In vitro ultrasonic lithotripsy is a new approach for the treatment of stones, it uses the powerful impact of ultrasonic echo to crush the body of calculus. Fiber-optic AE sensor used in medical ultrasonic lithotripsy has been productized, for example, the fiber-opticendoscope has been launched. Fiber-opticendoscopes can be used to detect ultrasonic shock waves, and the endoscopic probe made of fiber optics can be small to the order of microns. I is more convenient to detect.

The composite material undergoes the stages such as matrix cracking, fiber/matrix interface debonding, fiber breakage and fiber extraction when it bears alternating load. The abundant AE signals are generated at these stages, and the AE signal can be judged by the fiber AE sensor with the different damage forms and locations. Intelligent composite materials can not only perceive environmental changes, identify important stimuli, but can also make response to it. At present, the United States and Japan are all engaged in the design of intelligent composite structure and 
researches of active non-destructive testing. Fiber-optic AE sensor features small size, light weight, and can be adapt to the harsh environment; it is easy to form a distributed form, which makes it the preferred choice for the manufacture of intelligent composite materials.

The fiber-optic AE sensor can solve the problems like narrow wave band of traditional piezoelectric AE sensor, poor resistance to electromagnetic interference, etc. and thecoupling agent used for detecting the piezoelectric sensor changes the border state on surface of the object detected or leads to surface damage, whilefiber-optic AE sensorwould avoid such a situation, as there will no coupling agent be used, therefore, fiber-optic AE sensor technology has become a new orientation for $\mathrm{AE}$ detection and research. The use offiber-optic AE sensor allows early prediction of product defects, andthere are also the reports on practical engineering, including pressure pipe leak detection, tool health monitoring system, power equipment operation monitoring, etc.

For civil engineering, fiber-optics sensors are widely used in structural health monitoring of civil engineering, such as for bridges and dams. The fiber-optics sensor is affixed to the metal, concrete surface or buried in the building, which can perform real-time continuous monitoring of the building's stress, strain, crack initiation and expansion. In addition, the fiber-optic AE detection technology can also be used for acoustic emission research of rock material rupture.

\section{Problems and development trend of distributed fiber opticsacoustic sensing technology}

1) Poor vibration resistance: As the fiber optic sensor is extremely sensitive to vibration, so that small environmental vibration can also cause changes in the system signal, which is the main obstacle to the practical application of fiber optic AE sensor.

2) Light source fluctuation, loss of light intensity, phase delay, inconsistent polarization state and other factors will lead to instability of the output signal, so it is need to seek a variety of accurate phase compensation, polarization control, temperature compensation and other technologies.

3) Manufacturing process is not well-improved, and the productization and standardization are unavailable.

1) All-fiber miniaturization: It is popular that the sensor head consists of fiber optics and use only a fiber. All-fiber head is small and works reliably. Since the fusion loss among current fibersis $0.1 \mathrm{~dB}$ or so, this loss does not affect the normal work of the probe. At present, the bonding technologybetween the fibers and fiber end polishing, coating and other related technologies are in the study.

2)Multi-parameter real-timetransformation: A sensor can measure a number of parameters at the same time, which can reduce the number of components of the measurement device, but also to avoid the interactions between the sensors, therefore, real-time monitoring of multi-parameterbecome a hotspot of research. At present, many researchers have researched the fiber grating sensors which can measure temperature, stress and strain simultaneously, and have obtained some achievements.

3) High-precision practicalutilization: In the research process of the fiber-optics sensor, all components are linear idealized, and there're a certain gap with the practical application. Therefore, the non-linear study in the optical channel and the increase of dynamic range of actual detection are the basis for practical utilization.

4) Array form, networking, and easy to form a distributed detection system: A distributed detection system can greatly improve the detection efficiency, save detection costs, time 
manpower and resources. The use of wireless transmission and network for remote monitoring can also offer tremendous convenience for the real-time detection under special environment.

5) Enhancement of AE-source accurate positioning and recognition: Since AEsource positioning accuracy directly affect the follow-up maintenance and repair efficiency, therefore, it is extremelyimportant to the detection instruments for $\mathrm{AE}$ source high-precision positioning and damage pattern recognition.

\section{Conclusions:}

Distributed fiber optics sensing is a technology with promising application foreground. In fiber optical sensors, distributed fiber optical sensors are the most promising. It can perform the real-time, online and dynamic monitoring of the damage of concrete structures, while accurately evaluate the development of the cracks in the structure. In the civil engineering, where the distributed fiber optical sensors are successfully used, it can save the cost of detection,giving the huge economic benefits; also, it further enhance the measurement accuracy, and guarantee people's lives in a more reliable way.

\section{References}

[1] Qu Dandan.Study on Distributed Fiber optics Acoustic Emission Sensors [C]. Harbin: Harbin Engineering University, 2013.

[2] Zhao Jianghai. Advances in Fiber optics Acoustic Emission Sensing Technology [J]. Xi'an: Northwestern Polytechnical University, 2007.

[3]MA Yundong.Study on Sagnac Fiber Acoustic Emission Sensor [C]. Daqing: Equipment Manufacturing Technology, Issue 7, 2015.

[4] Research Status and Development of Fiber Optic Acoustic Emission Sensors [C]. Taiyuan: School of Information and Communication Engineering, North University of China, 030051. 